\title{
Difference harmonic generation due to spin-flip transitions in an asymmetric quantum well
}

\author{
A.V. Korovin \\ Institute of Semiconductor Physics, NAS of Ukraine, Kiev, 03028, Ukraine \\ E-mail: avkorovin@mailru.com
}

\begin{abstract}
The difference harmonic generation in an asymmetric quantum well based on AlSb/ $\mathrm{InSb}$ heterostructures has been calculated. The intersubband electron spin-flip transitions are analyzed in the framework of the three-band Kane model. Numerical results for the spectral dependencies of a nonlinear susceptibility under of a double resonance conditions for $\mathrm{CO}_{2}$ laser have been obtained.
\end{abstract}

Keywords: spin-flip transitions, asymetric quantum well, nonlinear susceptibility.

Paper received 01.12.00; revised manuscript received 03.01.01; accepted for publication 16.02.01.

\section{Introduction}

Currently, the nonlinear effects in low-dimensional structures are studied intensively. There are «up-conversion» (generation of the second [1] and higher order harmonics [2]) and «down-conversion» (difference harmonic generation [3]) processes among the basic directions both experimental, and theoretical researches of nonlinear effects, which are used mainly for generation of far infrared radiation. However, these works are devoted to the intersubband resonant transitions in the quantum wells (QW), which are perturbed by perpendicular to the 2Dplane component of an electrical field. Contrary to such transitions without change of the electron spin, the difference harmonic generation in an asymmetric heterostructures with non-degenerated by spin energy spectrum, where in-plane electrical field perturbs spin-flip electron transitions (the combined transitions [4]), has been considered in this paper.

The mechanism of spin-flip transitions was considered more than twenty years ago, at the research of inversion layers of a narrow-gap materials [5]. The active study of a narrow-gap QW based on InAs with appreciable a spin-splitting of an energy spectrum [6], which will be carried out recently, stimulates consideration of the linear and nonlinear responses in such structures at the account of the combined transitions. Such spin-splitting energy spectrum appears both due to the asymmetry of limiting potential $[7,8]$ and due to the asymmetry of boundary conditions at the heterojunction [8-11] and is de- scribed by linear to the electronic momentum $\mathbf{p}$ of the phenomenological contribution into the Hamiltonian [12].

Below, the difference harmonic generation in an asymmetric QW based on InAs is analyzed. Analysis is based on the three-band Kane model with a homogeneous electrical field, which is simply described by in-well potential. The influence of collisions is phenomenologically taken into account without the exchange renormalization of an energy spectrum.

\section{Second order response}

The basic expression for third order tensor of a nonlinear susceptibility that describe the difference harmonic generation has a form [13]:

$$
\begin{aligned}
& \chi_{\alpha \beta \gamma}^{\Delta \omega}=\frac{i e^{3}}{\omega_{1} \omega_{2} \Delta \omega L^{2}} \sum_{v v^{\prime} v^{\prime \prime}}\left(f_{v}-f_{v^{\prime}}\right) \times \\
& \times\left\{\frac{\left\langle v^{\prime}\left|\hat{V}_{\gamma}\right| v\right\rangle \Theta_{\alpha \beta}\left(v, v^{\prime}, v^{\prime \prime}\right)}{\varepsilon_{v^{\prime} v}+\hbar \omega_{2}-i \lambda}+\frac{\left\langle v^{\prime}\left|\hat{V}_{\beta}\right| v\right\rangle \Theta_{\alpha \gamma}\left(v, v^{\prime}, v^{\prime \prime}\right)}{\varepsilon_{v^{\prime} v}-\hbar \omega_{1}-i \lambda}\right\}
\end{aligned}
$$

Here, used is the basis of an eigenstate problem $\hat{h} v=$ $=\varepsilon_{v}|v\rangle$, where $\hat{h}$ is the Hamiltonian of Kane's model, $|v\rangle$ and $\varepsilon_{v}$ are an eigenvector and an energy for $v^{\text {th }}$ state; $\omega_{1}$ and $\omega_{2}$ are the frequencies of external laser pump ( $\left.\Delta \omega \equiv \omega_{1}-\omega_{2}\right), L^{2}$ is the normalization area, $f_{v}$ is the distribution function over $v^{\text {th }}$ state (a zero-temperature condition has been used for numerical estimations, so 


\section{A.V. Korovin: Difference harmonic generation due to spin-flip transitions in ...}

that $f_{v} \approx \theta\left(\varepsilon_{F}-\varepsilon_{v}\right)$, where $\varepsilon_{F}$ is the Fermi energy). Like Eq. (1) the following factor was introduced

$$
\begin{aligned}
& \Theta_{\alpha \beta}\left(v, v^{\prime}, v^{\prime \prime}\right)=\frac{\left\langle v\left|\hat{V}_{\alpha}\right| v^{\prime \prime}\right\rangle\left\langle v^{\prime \prime}\left|\hat{V}_{\beta}\right| v^{\prime}\right\rangle}{\varepsilon_{v^{\prime \prime} v}-\hbar \Delta \omega-i \lambda}- \\
& -\frac{\left\langle v\left|\hat{V}_{\beta}\right| v^{\prime \prime}\right\rangle\left\langle v^{\prime \prime}\left|\hat{V}_{\alpha}\right| v^{\prime}\right\rangle}{\varepsilon_{v^{\prime} v^{\prime \prime}}-\hbar \Delta \omega-i \lambda} .
\end{aligned}
$$

with phenomenologically introduced the broadening energy, $\lambda$, which independent of the quantum numbers; $\alpha, \beta$ and $\gamma$ are the Cartesian coordinate indexes.

The heterojunction AlSb-InAs is characterized by big break of the $c$-band, that lead to strong electron localization inside QW. Therefore, for the bottom quantum levels, it is possible to neglect the penetration of the wave function into the barrier range (the approximation of indefinite barriers).

The stationary electronic states in a narrow-gap heterostructures are described by the three-band Kane Hamiltonian [14]. In the assumption of the small contribution from another bands, and also, that inverse heavy hole effective mass is equal to zero, Kane's Hamiltonian inside the well range can be written as

$$
\hat{h}=\hat{\varepsilon}_{z}+(\hat{\mathbf{v}} \cdot \hat{\mathbf{p}})
$$

Here $\hat{\mathbf{p}}$ is the kinematics momentum (in the $\mathbf{p} z$ representation $\hat{\mathbf{p}} \equiv\left\{\mathbf{p}, \hat{p}_{z}\right\}$, where $\mathbf{p}$ is the $2 \mathrm{D}$-momentum, $\hat{p}_{z}$ is the operator of momentum along OZ); $\hat{\varepsilon}_{z}$ is the diagonal matrix of the energy extrema $\left(\varepsilon_{c z}, \varepsilon_{h z}\right.$ and $\varepsilon_{l z}$ are the extrema of the electrons, heavy and light holes, accordingly) and $\hat{\mathbf{v}}$ is the matrix elements of interband velocity, which described by the Hermitian $6 \times 6$ matrix, with nonzero elements

$$
\begin{aligned}
& \mathrm{v}_{13}^{x}=\mathrm{v}_{26}^{x}=\frac{\mathscr{P}}{\sqrt{2}}, \mathrm{v}_{15}^{x}=-\mathrm{v}_{24}^{x}=-\frac{\mathscr{P}}{\sqrt{6}}, \mathrm{v}_{13}^{y}=-\mathrm{v}_{26}^{y}=\frac{i \mathscr{P}}{\sqrt{2}}, \\
& \mathrm{v}_{15}^{y}=\mathrm{v}_{24}^{y}=\frac{i \mathscr{P}}{\sqrt{6}}, \mathrm{v}_{14}^{z}=\mathrm{v}_{25}^{z}=\mathscr{P} \sqrt{\frac{2}{3}},
\end{aligned}
$$

where $\mathscr{P}$ is the characteristic interband velocity for Kane's model, and the wave function is represented by six-rows column-matrix with components $\psi_{\mathbf{p} z}^{(1-6)}$. For simplicity sake we have neglected the strain effect due to lattice mismatch, and use $\varepsilon_{v z} \equiv \varepsilon_{h z}=\varepsilon_{l z}$, then after substitution Eq. (4) into Eq. (3), the eigenstate problem takes the following form
Here we introduce $P \equiv p_{x}+i p_{y}$, where $p_{x}$ and $p_{y}$ are the components of a $2 \mathrm{D}$-vector $\mathbf{p}$. For determination of electronic states in $c$-band, we except the components of wave function of a $v$-band $\psi_{\mathbf{p} z}^{(3-6)}$ from the eigenstate problem (5), so that we obtain the effective Shrodinger equation for the spinor of a $c$-band $\Psi_{\mathbf{p} z}$ with components $\psi_{\mathbf{p} z}^{(1-2)}$ :

$\left\{\varepsilon_{c z}-\varepsilon+\frac{p^{2}}{2 m_{z}}+\hat{p}_{z} \frac{1}{2 m_{z}} \hat{p}_{z}+\mathbf{v}_{z}[\mathbf{p} \times \hat{\boldsymbol{\sigma}}]\right\} \Psi_{\mathbf{p} z}=0$,

where $\hat{\boldsymbol{\sigma}}$ is the vector made on Pauli matrixes, the last member in Eq. (6) describes spin-orbit interaction for 2Delectrons. Besides, we have introduced in Eq. (6), dependent on $z$ effective mass, $m_{z}$, and characteristic «spin» velocity, $\mathbf{v}_{\mathrm{z}}$, directed along the growth axis $(\mathrm{OZ})$, in accordance to ratios:

$m_{z}=-m \frac{\varepsilon_{v z}-\varepsilon}{\varepsilon_{g}}, \mathrm{v}_{z}=\frac{\hbar \varepsilon_{g}}{4 m} \frac{\partial \varepsilon_{v z} / \partial z}{\left(\varepsilon_{v z}-\varepsilon\right)^{2}}$

where $m \equiv 4 \mathscr{P}^{2} /\left(3 \varepsilon_{g}\right)$ is the bulk effective mass, $\varepsilon_{g}$ is the gap energy for InAs. The energy extrema of $c$ - and $v$ bands in QW (see the band diagram in Fig. 1) have the following form

$\varepsilon_{c z}=U_{z} ; \varepsilon_{v z}=-\varepsilon_{g}+U_{z}$,

here $U_{z}$ is the in-well potential, for numerical estimations presented below, we determine $U_{z}$ for undoped $\mathrm{QW}$, as $U_{z} \approx|e| F_{\perp} z$, where $F_{\perp}$ is the external transverse homogeneous electrical field (for strong doped structures, $F_{\perp}$ will be introduced as an average mean of a self-consistent field).

We consider weak electric fields. It means that, then $\varepsilon_{g} \gg U_{z}$ Eq. (6) should be written as

$\left\{U_{z}-\varepsilon+\frac{p^{2}+\hat{p}_{z}^{2}}{2 m_{\varepsilon}}+\hat{\boldsymbol{\sigma}}\left[\mathbf{v}_{\varepsilon} \times \mathbf{p}\right]\right\} \Psi_{\mathbf{p} z}=0$

Here the «effective mass» and characteristic «spin»" velocity (4) depend on energy of the quantum levels:

$$
m_{\varepsilon}=m\left(1+\varepsilon / \varepsilon_{g}\right), \mathrm{v}_{\varepsilon}=\frac{|e| F_{\perp} \hbar}{4 m \varepsilon_{g}\left(1+\varepsilon / \varepsilon_{g}\right)^{2}} .
$$

$$
\left(\begin{array}{cccccc}
\varepsilon_{c z}-\varepsilon & 0 & (\mathscr{P} / \sqrt{2}) P & \sqrt{2 / 3} \mathscr{P} \hat{p}_{z} & (\mathscr{P} / \sqrt{6}) P^{*} & 0 \\
0 & \varepsilon_{c z}-\varepsilon & 0 & (\mathscr{P} / \sqrt{6}) P & \sqrt{2 / 3} \mathscr{P} \hat{p}_{z} & (\mathscr{P} / \sqrt{2}) P^{*} \\
(\mathscr{P} / \sqrt{2}) P^{*} & 0 & \varepsilon_{v z}-\varepsilon & 0 & 0 & 0 \\
\sqrt{2 / 3} \mathscr{P} \hat{p}_{z} & (\mathscr{P} / \sqrt{6}) P^{*} & 0 & \varepsilon_{v z}-\varepsilon & 0 & 0 \\
(\mathscr{P} / \sqrt{6}) P & \sqrt{2 / 3} \mathscr{P} \hat{p}_{z} & 0 & 0 & \varepsilon_{v z}-\varepsilon & 0 \\
0 & (\mathscr{P} / \sqrt{2}) P & 0 & 0 & 0 & \varepsilon_{v z}-\varepsilon
\end{array}\right)\left(\begin{array}{c}
\psi_{p z}^{(1)} \\
\psi_{p z}^{(2)} \\
\psi_{p z}^{(3)} \\
\psi_{p z}^{(4)} \\
\psi_{p z}^{(5)} \\
\psi_{p z}^{(6)}
\end{array}\right)=0
$$


A.V. Korovin: Difference harmonic generation due to spin-flip transitions in ...

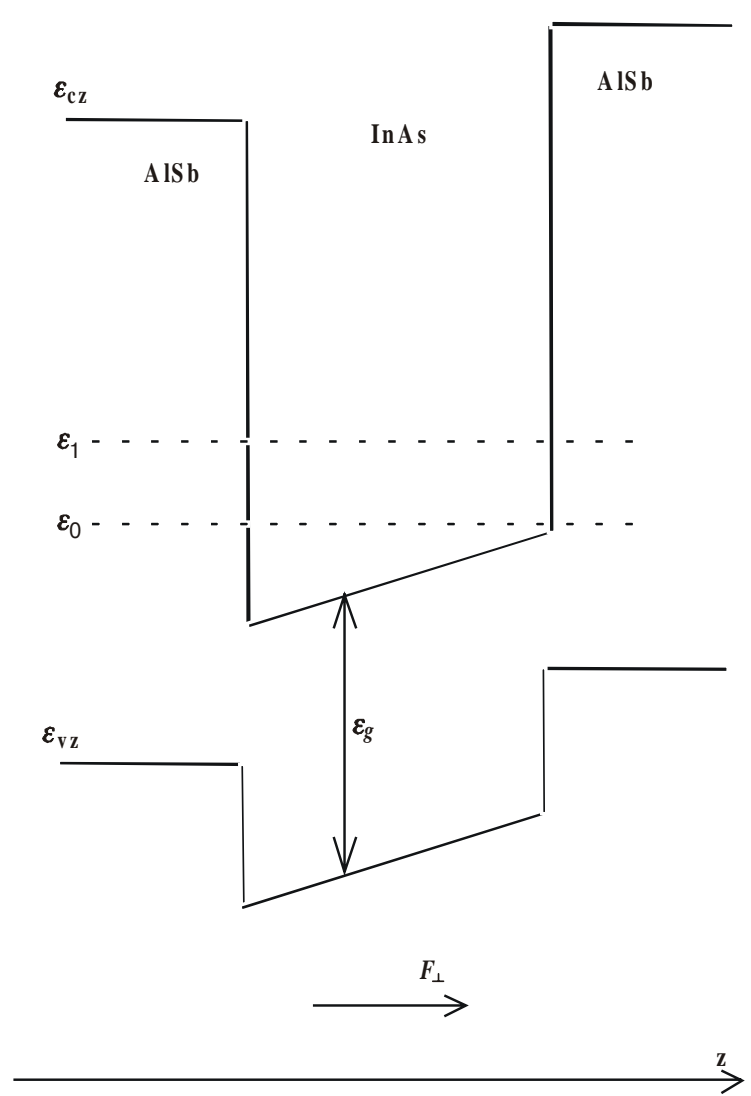

a)

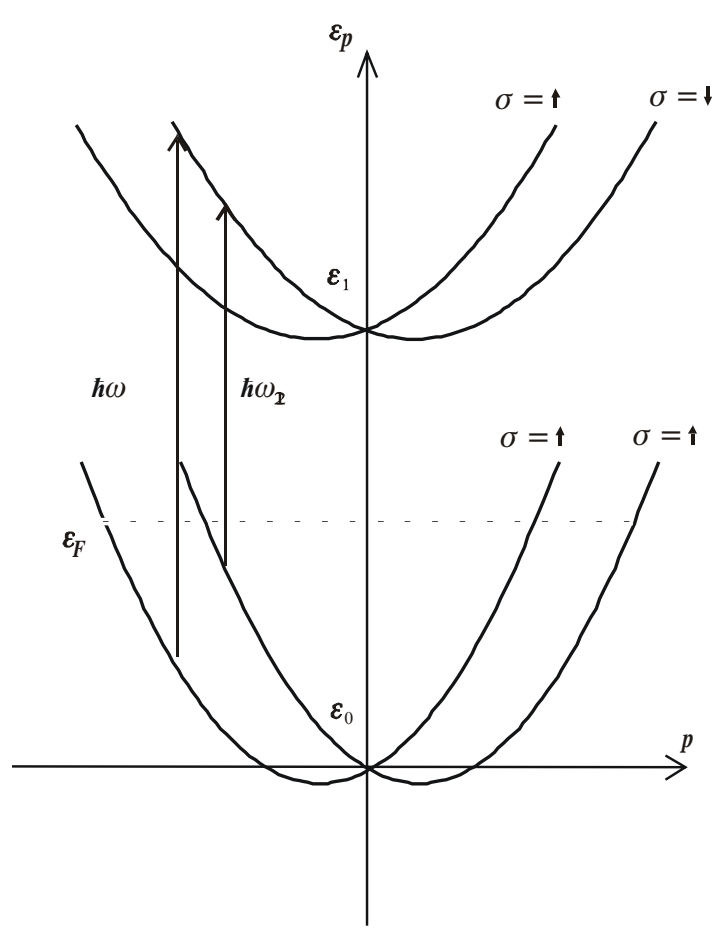

b)

Fig. 1. a) The schematic band diagram for AlSb-InAs-AlSb quantum well;

b) $2 \mathrm{D}$ electrons dispersion law for heterostructure in a homogeneous transverse electrical field $F_{\perp}\left(\hbar \omega_{1}\right.$ and $\hbar \omega_{2}$ are frequencies of of pumping)

Separating variables in the Shroedinger Eq. (9) and using unitary transformation [15] for diagonalizing the spin-orbit interaction term in Eq. (9), we finally obtain for $c$-band spinor:

$\Psi_{n \mathbf{p} z}^{\sigma}=\frac{\varphi_{n z}}{\sqrt{2}}\left[1+i \frac{(\boldsymbol{\sigma} \cdot \mathbf{p})}{p}\right]|\sigma\rangle$,

where $\varphi_{n z}$ is the eigenfunction for the eigenstate problem without spin-orbital interaction: $\left(\hat{p}_{z}^{2} /\left(2 m_{\varepsilon_{n}}\right)+\right.$ $\left.|e| F_{\perp} z\right) \varphi_{n z}=\varepsilon_{n} \varphi_{n z}$, where $\varepsilon_{n}$ is the energy of quantum levels (in our case we consider levels with $n=0,1$ only), $|\sigma\rangle-$ spin functions $\left(\hat{\sigma}_{z}|\sigma\rangle=\sigma|\sigma\rangle\right.$, where $\left.\sigma \equiv \pm 1\right)$. Taking into account Eq. (11), the energy dispersion law takes a form:

$\varepsilon_{n \mathbf{p}}^{\sigma}=\varepsilon_{n}+\frac{p^{2}}{2 m_{n}}+\sigma v_{n} p$,

where the effective mass and the characteristic «spin» velocity (10) are determined by meaning of the quantum level energy $\varepsilon_{n}$ (so that $m_{\varepsilon_{n}} \equiv m_{n}$ and $v_{\varepsilon_{n}} \equiv v_{n}$ ).
Using spinor function (11) and taking into account a component of wave function of $v$-band $\psi_{\mathbf{p} z}^{(3-6)}$ (5), the undiagonal matrix elements of the velocity operator (2) take a form:

$$
\left\langle\sigma n\left|\hat{V}_{x, y}\right| n^{\prime} \sigma^{\prime}\right\rangle_{\mathbf{p}}=\sigma^{\prime}\left\{\frac{i P_{n n^{\prime}}^{\perp}}{4}\left(\frac{1}{m_{n}}-\frac{1}{m_{n^{\prime}}}\right)-\delta_{n n^{\prime} v_{n}}\right\} \frac{p_{x, y}}{p},
$$

$\left\langle\sigma n\left|\hat{V}_{z}\right| n^{\prime} \sigma^{\prime}\right\rangle_{\mathbf{p}}=\delta_{\sigma \sigma^{\prime}} \frac{P_{n n^{\prime}}^{\perp}}{2}\left(\frac{1}{m_{n}}+\frac{1}{m_{n^{\prime}}}\right)$,

where $P_{n n^{\prime}}^{\perp} \equiv\left\langle n\left|\hat{p}_{z}\right| n^{\prime}\right\rangle$ is the matrix elements of the operator of transverse to QW plane momentum (for unlimited rectangle $\mathrm{QW}$, we can estimate $P_{10}^{\perp}$ as $\left.8 i \hbar /(3 d)\right)$. The matrix elements of longitudinal velocity (13) are determined by components of $2 \mathrm{D}$-vector $\mathbf{p}$, then due to symmetry, non-zero components of a nonlinear susceptibility will be $\chi_{z \alpha \alpha}, \chi_{\alpha z \alpha}$ and $\chi_{\alpha \alpha z}$ only, here $\alpha$ is a projection to any in-plane axis. The matrix elements of transverse velocity describe the transitions between different quantum levels without spin-flip process only.

Using a double resonance condition, when $\hbar \omega_{1,2} \approx \varepsilon_{1 \mathbf{p}}^{\sigma}-\varepsilon_{0 \mathbf{p}}^{\sigma^{\prime}}$ and $\hbar \Delta \omega \approx \varepsilon_{n \mathbf{p}}^{\sigma}-\varepsilon_{n \mathbf{p}}^{\sigma^{\prime}}$, after substitution 


\section{A.V. Korovin: Difference harmonic generation due to spin-flip transitions in ...}

of matrix elements $(13,14)$ into Eq. (1) and simple, but cumbersome transformations, we obtain:

$$
\begin{aligned}
& \chi_{\alpha \alpha z}^{\Delta \omega}=\frac{|e|^{3} V^{2}}{8 \omega_{1} \omega_{2} \Delta \omega} \sum_{\sigma} \int \frac{d \mathbf{p}}{(2 \pi \hbar)^{2}}\left(\frac{p_{\alpha}}{p}\right)^{2} \theta\left(\varepsilon_{F}-p^{2} /\left(2 m_{0}\right)-\right. \\
& \left.-\sigma v_{0} p\right) \times\left\{\frac{\frac{v_{1}}{-2 \sigma v_{1} p-\hbar \Delta \omega-i \lambda}-\frac{v_{0}}{2 \sigma v_{0} p-\hbar \Delta \omega-i \lambda}}{p^{2} / \mu+\sigma\left(v_{0}-v_{1}\right) p-\delta \varepsilon-\hbar \Delta \omega / 2-i \lambda}+\right. \\
& \left.\frac{\frac{v_{1}}{2 \sigma v_{1} p+\hbar \Delta \omega+i \lambda}-\frac{v_{0}}{2 \sigma v_{0} p+\hbar \Delta \omega+i \lambda}}{p^{2} / \mu+\sigma\left(v_{0}+v_{1}\right) p-\delta \varepsilon+\hbar \Delta \omega / 2+i \lambda}\right\},
\end{aligned}
$$

here introduced $V^{2} \equiv P_{10}^{\perp} P_{01}^{\perp}\left(1 / m_{0}{ }^{2}-1 / m_{1}{ }^{2}\right), 1 / \mu \equiv$ $\equiv 1 / m_{0}-1 / m_{1}$, and $\delta \varepsilon \equiv \Delta \varepsilon-\left(\hbar \omega_{1}+\hbar \omega_{2}\right) / 2$ is the detuning energy, and $\Delta \varepsilon \equiv \varepsilon_{1}-\varepsilon_{0} ; \delta \varepsilon \equiv \hbar \omega_{2}-\Delta \varepsilon$ is the energy distance between quantum levels. Besides, in Eq. (15) we consider occupied spin-splitted bottom levels only, and the summation on all 2D-momenta is replaced by integration. Moreover, $\chi_{\alpha z \alpha}\left(\omega_{1}, \omega_{2}\right)=-\chi_{\alpha \alpha z}\left(\omega_{2}, \omega_{1}\right)^{*}$, and $\chi_{z \alpha \alpha}<\chi_{\alpha \alpha z}, \chi_{\alpha \alpha z}$, because $\chi_{z \alpha \alpha}$ do not satisfy double resonance conditions.

Finally, after integration, we rewrite Eq. (15) as:

$\chi_{\alpha \alpha z}^{\Delta \omega}(\delta \varepsilon, \hbar \Delta \omega)=\frac{\hbar|e|^{3} V^{2}}{32 \pi} \frac{\sum_{\sigma} I_{\delta \varepsilon, \hbar \Delta \omega}^{\sigma, \sigma}+\left(I_{\delta \varepsilon,-\hbar \Delta \omega}^{\sigma,-\sigma}\right)^{*}}{\left((\Delta \varepsilon-\delta \varepsilon)^{2}-\hbar \Delta \omega^{2} / 4\right) \hbar \Delta \omega}$

Here $p_{F}=\sqrt{2 m_{0} \varepsilon_{F}}$, and the introduced integral function has a form

$$
I_{\delta \varepsilon, \hbar \Delta \omega}^{\sigma_{0}, \sigma_{1}} \equiv \int_{0}^{p_{F}-\sigma_{0} m_{0} v_{0}} x d x \frac{\frac{v_{1}}{-2 \sigma_{1} v_{1} x-\hbar \Delta \omega-i \lambda}-\frac{v_{0}}{2 \sigma_{0} v_{0} x-\hbar \Delta \omega-i \lambda}}{x^{2} / \mu+\left(\sigma_{0} v_{0}-\sigma_{1} v_{1}\right) x-\delta \varepsilon-\hbar \Delta \omega / 2-i \lambda},
$$

Just after integration of the function (17), without nonresonant terms, we can rewrite (17) as

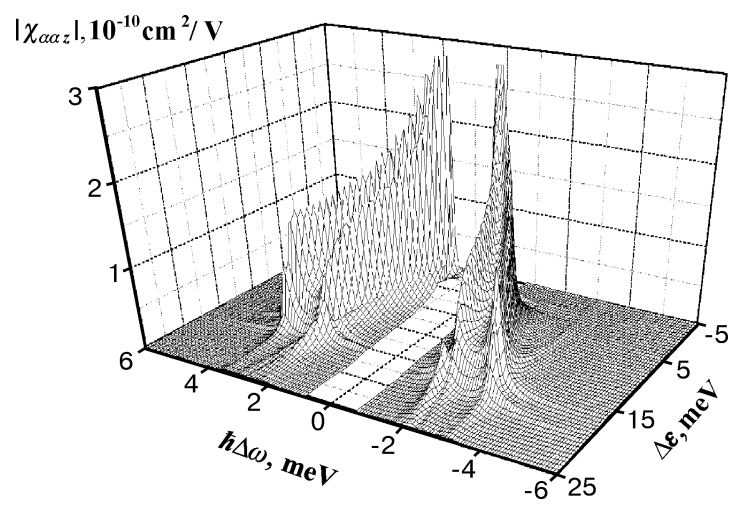

Fig. 2. Spectral dependence $\left|\chi_{\alpha \alpha z}\right|$ versus detuning energy $\delta \varepsilon$ and difference frequency $\hbar \Delta \omega(\lambda=0.1 \mathrm{meV})$.

\section{Numerical estimation}

The numerical estimations were obtained for AlSb-InAsAlSb QW, with $15 \mathrm{~nm}$ well widths. Other parameters used in the calculation are standard for bulk InAs: the gap energy is $\varepsilon_{g} \approx 0.5 \mathrm{eV}$, the effective mass is $m \approx 0.022 m_{e}$ ( $m_{e}$ is the free electron mass). These parameters give us distance between quantum levels of the order of $\Delta \varepsilon \approx 140 \mathrm{meV}$.

The spectral dependencies of absolute values $\left|\chi_{\alpha \alpha z}\right|$ versus a detuning energy $\delta \varepsilon$ and energy of the difference frequency $\hbar \Delta \omega$ at the broadening energy $\lambda=0.1 \mathrm{meV}$ are presented in Fig. 2. From function (18) it follows that double resonance conditions are satisfied when denominators in (18) are equal to zero, therefore, spectral dependency has multi-peak structure (two parabolic curves $\delta \varepsilon(\hbar \Delta \omega)$ with different sign at $v_{0}$ and $v_{1}$ in (18), where $\left.\delta \varepsilon<p_{F}{ }^{2} / \mu\right)$. As we consider weak external electrical fields (so that $\varepsilon_{g}>>U_{z}$ ), then, for the external electrical field $F_{\perp} \approx 70 \mathrm{kV} / \mathrm{cm}$, the resonance at the difference frequency arises at the following energies $|\hbar \Delta \omega| \approx 3 \mathrm{meV}$, $|\hbar \Delta \omega| \approx 2 \mathrm{meV}$ and $\delta \varepsilon \approx 16 \mathrm{meV}$, but these peaks are merged due to the non-zero broadening energy. Such spectral dependency at the broadening energy $\lambda=0.5 \mathrm{meV}$

$$
\begin{gathered}
I_{\delta \varepsilon, \hbar \Delta \omega}^{\sigma_{0}, \sigma_{1}} \equiv(\hbar \Delta \omega+i \lambda)\left[v_{1} \frac{\ln \left(1+\frac{2 \sigma_{1} v_{1} p}{\hbar \Delta \omega+i \lambda}\right)-\ln \sqrt{1-\frac{p^{2} / \mu+\left(\sigma_{0} v_{0}-\sigma_{1} v_{1}\right) p}{\delta \varepsilon+\hbar \Delta \omega / 2+i \lambda}}}{\frac{(\hbar \Delta \omega+i \lambda)^{2}}{\mu}+2 v_{1}\left(v_{1}-\sigma_{1} \sigma_{0} v_{0}\right)(\hbar \Delta \omega+i \lambda)-4 v_{1}^{2}(\delta \varepsilon+\hbar \Delta \omega / 2+i \lambda)}\right. \\
\left.-v_{0} \frac{\ln \left(1-\frac{2 \sigma_{0} v_{0} p}{\hbar \Delta \omega+i \lambda}\right)-\ln \sqrt{1-\frac{p^{2} / \mu+\left(\sigma_{0} v_{0}-\sigma_{1} v_{1}\right) p}{\delta \varepsilon+\hbar \Delta \omega / 2+i \lambda}}}{\frac{(\hbar \Delta \omega+i \lambda)^{2}}{\mu}+2 v_{0}\left(v_{0}-\sigma_{0} \sigma_{1} v_{1}\right)(\hbar \Delta \omega+i \lambda)-4 v_{0}^{2}(\delta \varepsilon+\hbar \Delta \omega / 2+i \lambda)}\right]
\end{gathered}
$$

where we introduce $p \equiv p_{F}-\sigma_{0} m_{0} \mathrm{v}_{0}$. 
A.V. Korovin: Difference harmonic generation due to spin-flip transitions in ...

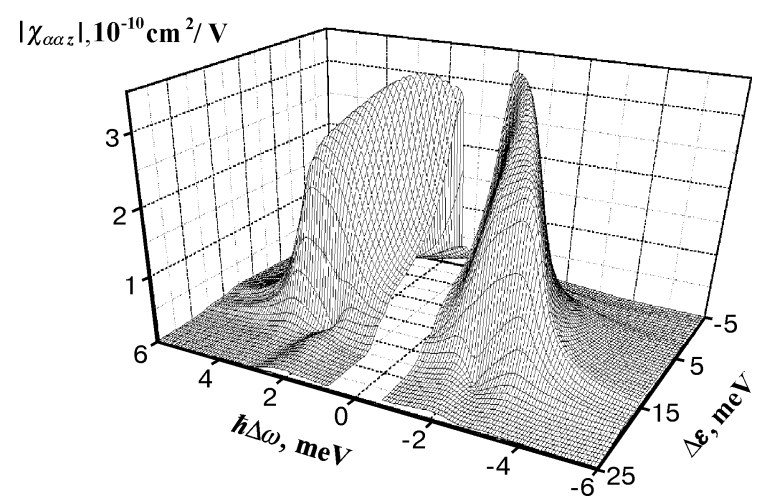

Fig. 3. Spectral dependence $\left|\chi_{\alpha \alpha z}\right|$ versus detuning energy $\delta \varepsilon$ and difference frequency $\hbar \Delta \omega(\lambda=0.5 \mathrm{meV})$.

is presented in Fig. 3. We did not consider energy range $|\hbar \Delta \omega|<\lambda$, because Eq. (1) is not satisfied, when the pump photon energy is closed to the broadening energy.

The absolute value $\chi_{\alpha \alpha_{z}}$, it's real and imaginary parts both as functions from detuning energy $\delta \varepsilon$ at fixed difference photon energy $\hbar \Delta \omega=5 \mathrm{meV}$ and as functions from difference photon energy $\hbar \Delta \omega$ at fixed energy of pump photon $\hbar \omega_{1}=130 \mathrm{meV}$ corresponding to $\mathrm{CO}_{2}$ laser, are shown in Fig. 4 and Fig. 5 (with the broadening energy $\lambda=0.5 \mathrm{meV}$ ).

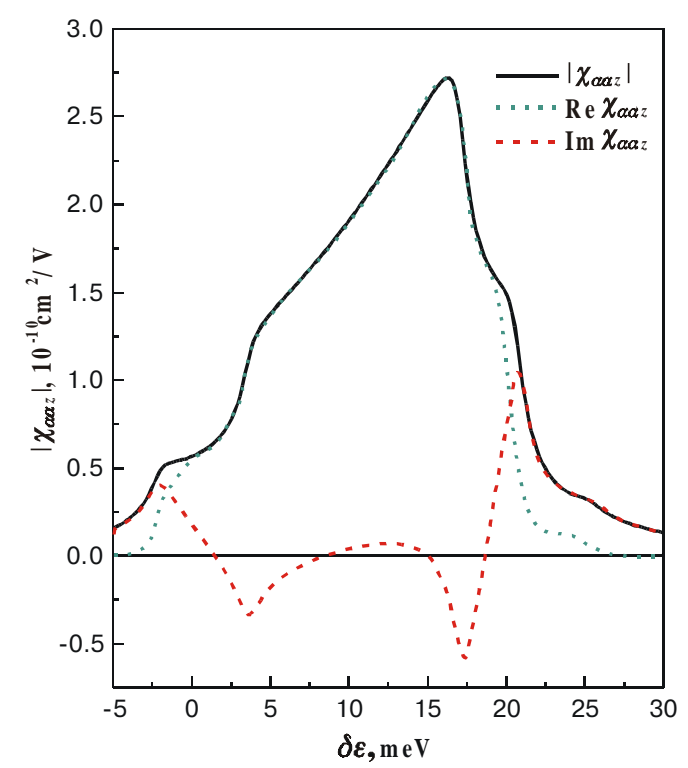

Fig. 4. The spectral dependence of absolute value, real and image part of $\left|\chi_{\alpha \alpha z}\right|$ versus to detuning energy $\delta \varepsilon$ at fixed difference frequency photon energy $\hbar \Delta \omega=5 \mathrm{meV}$.

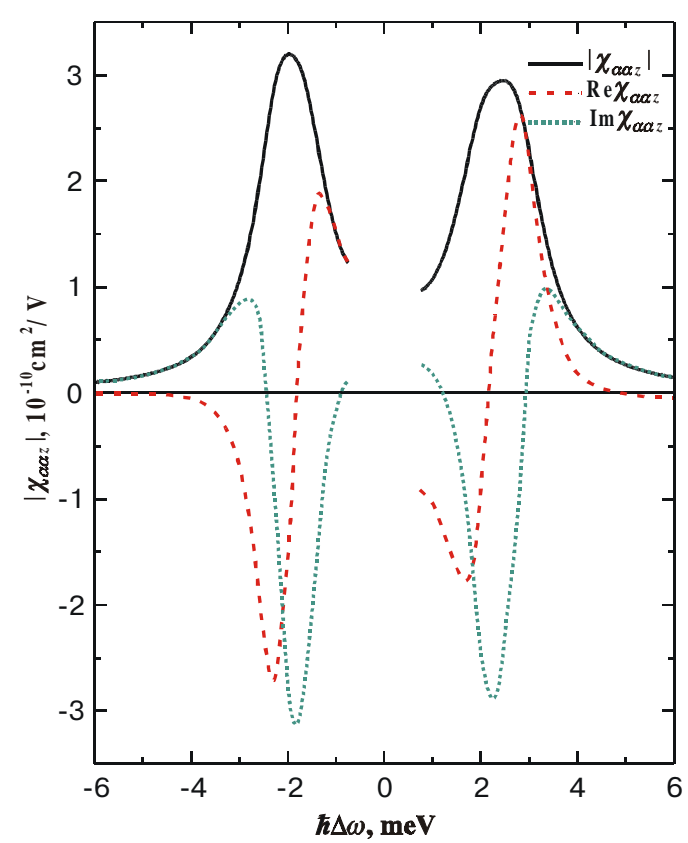

Fig. 5. The spectral dependence of absolute value, real and image part of $\left|\chi_{\alpha \alpha z}\right|$ versus to differece energy $\hbar \Delta \omega$ at fixed pump photon energy $\hbar \Delta \omega_{1}=130 \mathrm{meV}$.

\section{Conclusions}

In this work based on the three-band Kane model, the opportunity of application of the combined transitions for the difference harmonic generation in an asymmetric heterostructures with non-degenerated by spin energy spectrum has been analyzed. The analysis showed that such structures are possible to be used for effective transformation Mid-IR radiation into $\mathrm{THz}$ radiation. However, the obtained value of a nonlinear susceptibility is significant less than in tunnel-coupled and «step-like» $\mathrm{QW}$, but is commensurable with such value for QWs based on thickness metal films [3].

Let's consider of the basic approximation used in this work. At calculations of the stationary states in InAs we did not took into account the contribution from cubic on p term (which is due to inversion asymmetry of bulk material [16] formed QW, the contribution from such members is discussed in [17], too). The experimental data for various heterostructures based on InAs [18] clearly show the dominant linear to $\mathbf{p}$ contribution of the terms in spinorbit interaction in narrow-gap heterostructures. The relative value of the contributions from in-well potential and potential jump in heterojunction experimentally was not investigated, but theoretical estimations gave the identical order of these contributions [19]. Phenomenologically introduced broadening energy is widely distributed and satisfies to the purpose for estimation of efficiency of generation. The influence of electron-electron interaction was not considered, because this influence is negligible for InAs at weak 2D electron concentration (around $4 \cdot 10^{11} \mathrm{~cm}^{-2}$ ). 


\section{A.V. Korovin: Difference harmonic generation due to spin-flip transitions in ...}

\section{Acknowledgments}

The author thanks participants of the seminar of the Department of theoretical physics of Institute of Semiconductor Physics for their useful remarks and advices.

\section{References}

1. W. W. Bewley, G. L. Felix, J. J. Plombon, M. S. Sherwin, M. Sandaram, P. F. Hopkins and A. C. Gossard, Phys. Rev. B 48, 2376-2390 (1993); J. B. Kurgin, Appl. Phys. Lett. 67, 1113-1115 (1995)

2. Y. Huang, C. Lien, Phys. Low. -Dim. Str. , 1-52 (1995); J. B. Kurgin, S. Li Appl. Phys. A 53, 523-534 (1991).

3. C. Sirtori, F. Capasso, J. Faist, L. N. Pfeiffer and K. W. West, Appl. Phys. Lett. 67, 445-447 (1994); A. V. Korovin, F. T. Vasko, Appl. Phys. B 68, 355 (1999).

4. E.I. Rashba, Uspekhi Phiz. Nauk 84, 557 (1964).

5. W. Beinvogl, F. Koch, Sol. St. Commun. 24, 687 (1977); F.T. Vasko and N.A. Prima, Sov. Phys. - Solid State 21, 1734 (1979).

6. J.P. Heida, B. J. Van Wees, J. J. Kuipers, T. M. Klapwijk, and G. Borghs, Phys. Rev. B 57, 11911 (1998); A.J. Nitta, T. Akazaki, H. Takayanagi, and T. Enoki, Phys. Rev. Lett. 78 , 1335 (1997); J. Luo, H. Munekata, F. F. Fang, and P. J. Stiles, Phys. Rev. B 41, 7685 (1990).

7. F.J. Ohkawa and Y. Uemura, J. Phys. Soc. Jpn. 37, 1325 (1974); Y. Takada. K. Arai, N. Uchimura, and Y. Uemura, J. Phys. Soc. Jpn. 49, 1851 (1980).
8. F.T. Vasko. JETP Lett. 30, 541 (1979).

9. R.A. Suris and A.B. Sokolovski, Sov. Phys. Semicond. 21, 529 (1987)

10. G. Bastard, J.A. Brum, and R. Ferreira, Solid State Phys. 44, 229 (1991).

11. P. Pfeiffer and W. Zawadzki, Phys. Rev. B 52, R14332 (1995).

12. Yu.A. Buchkov and E.I. Rashba, JETP Lett. 39, 78 (1984); J. Phys. C. 17, 6039 (1984).

13. R. W. Boyd, Nonlinear Optics (Academic, Boston, 1992); Y. R. Shen, The princiles of Nonlinear Optics (Wiley, New York, 1984)

14. F. T. Vasko and A. V. Kuznetsov, Electron States and Optical Transitions in Semiconductor Heterostructures (Springer, N. Y. 1998).

15. F. T. Vasko, O. Keller, Phys. Rev. B 58, 15666 (1998).

16. E.I. Rashba, Sov. Phys. - Solid State, 2, 1109 (1960) [Fiz. Tverd. Tela.] 2, 1224 (1960).

17. M.I. Dyakonov and T. M. Kocharovski, Fiz.Techn. Poluprovodn. 20, 178 (1986) [Sov. Phys. - Semicond. 20, N1 (1986)]; P. Eppenga and M.F.H. Schuurmans, Phys. Rev. B 37, 10923 (1988)

18. J. Nitta, T. Akazaki, H. Takayanagi, and T. Enoki, Phys. Rev. Lett. 78, 1335 (1997); J.P. Heida, B.J. van Wees, T.M. Klapwijk, and G. Borghs. 23rd Int. Conf. on the Physics of Semiconductors, p.2467. Ed. by M. Scheffler and R. Zimmermann (World Scientific, 1996); J. Luo, H. Munekata, F.F. Fang, and P.J. Stiles, Phys. Rev. B 41, 7685 (1990).

19. E.A. de Andrada e Silva, G.C. La Rocca, and F. Bassani, Phys. Rev. B 50, 8523 (1994); Phys. Rev. B 55, 16293 (1997). 